\title{
ORIGINAL
}

\section{SATISFACCIÓN PARENTAL CON EL APOYO RECIBIDO DURANTE LA PRIMERA INFANCIA POR EL EQUIPO DE PEDIATRÍA: DESARROLLO Y VALIDACIÓN DE UN CUESTIONARIO(*)}

\author{
Enrique Callejas Castro (1), Sonia Byrne (1) y María José Rodrigo López (1) \\ (1) Universidad de La Laguna. Santa Cruz de Tenerife. España. \\ Los autores declaran que no existe conflicto de intereses. \\ (*) Financiación: Este trabajo ha sido subvencionado por el Ministerio de Educación, Cultura y Deporte a través de una ayuda de \\ Formación de Profesorado Universitario [referencia FPU16/01039].
}

\section{RESUMEN}

Fundamentos: La Estrategia de Promoción de la Salud y Prevención de la Enfermedad del Sistema Nacional de Salud incluye una línea de prevención destinada a la infancia de cero a tres años que promueve el apoyo a las figuras parentales en la atención primaria. Este artículo describe el desarrollo y la validación de un cuestionario que evalúa la satisfacción de las figuras parentales con el apoyo recibido por el equipo de pediatría.

Métodos: Participaron 226 figuras parentales con hijos/as entre 0 y 36 meses, que eran usuarios del Servicio Canario de Salud. Los datos se recogieron durante los años 2018 y 2019. Para la validación del instrumento, se realizó un análisis factorial mediante un Modelo de Ecuaciones Estructurales Exploratorio con rotación oblimin, y el método de estimación de ponderación de mínimos cuadrados para su confirmación. Además, se analizaron las diferencias individuales según las variables sociodemográficas, y la salud infantil mediante ANOVAs.

Resultados: Se obtuvo una estructura factorial óptima de cuatro factores y 14 ítems, con una fiabilidad de $\alpha=0,84$, que recogen la "Adecuación del servicio", el "Descontento con el servicio", la relación de "Colaboración del profesional con la familia" y la promoción de la "Capacitación parental". El análisis del perfil sociodemográfico indicó que a mayor nivel educativo de las figuras parentales se constata una menor satisfacción con el servicio.

Conclusiones: Se ha elaborado una herramienta útil de evaluación para mejorar la calidad del servicio, de acuerdo con el Modelo Centrado en la Familia y el marco europeo de la Parentalidad Positiva, que cumple con las garantías psicométricas de validez y fiabilidad.

Palabras clave: Satisfacción, Apoyo parental, Pediatría, Atención primaria, Modelo centrado en la familia, Parentalidad positiva, Validación, Cuestionario.

\section{ABSTRACT}

Parental satisfaction with the support received during the early childhood by paediatric team: development and validation of a questionnaire

Background: The Prevention and Health Promotion Strategy of the Spanish National Health System has a prevention line aimed at early childhood in which parental support is promoted at primary care settings. This article describes the development and validation of a questionnaire that assesses parental satisfaction with the support received by the paediatric team.

Methods: Participants were 226 parental figures with children from birth to three, users of the Canarian Health System. Data was collected during 2018 and 2019. The factor structure was obtained by Exploratory Structural Equation Modeling (ESEM) with oblimin rotation, and the estimation method using the Weighted Least Squares with moving measurement window (WLSMW) for confirmatory purposes. Moreover, individual differences were analysed through sociodemographic variables and child health status by means of ANOVAs.

Results: The main results showed an optimal factorization of the construct involving a four-factor model and 14 items with a reliability of $\alpha=0.84$, dealing with 'Adequacy of the service', 'Discontent with the service', 'Collaboration with the family', and promotion of 'Parental capacity'. The sociodemographic profile analysis showed that a higher educational level was related to lower satisfaction with the service.

Conclusions: The questionnaire is an useful assessment tool to improve the quality of the service, according to the Family-Centred Model and the Positive Parenting European framework, which meets psychometric guarantees of validity and reliability.

Key words: Satisfaction, Parental support, Paediatrics, Primary care, Family-centred model, Positive parenting, Validation, Questionnaire. 


\section{INTRODUCCIÓN}

Una nueva visión del apoyo parental en los equipos de pediatría de atención primaria. La Estrategia de Promoción de la Salud y Prevención de la Enfermedad del Sistema Nacional de Salud ${ }^{(1)}$ incluye una línea de prevención temprana destinada a la infancia entre cero y tres años. En dicha línea se subraya la importancia de incluir el apoyo sistemático a las figuras parentales (padre, madre $u$ otros cuidadores principales) en el servicio de atención primaria por su papel central en el bienestar y en el desarrollo infantil. Una de las acciones que se ha patrocinado es el curso basado en evidencia "Ganar salud y bienestar de 0 a 3 años"(2). Se trata de una iniciativa novedosa de $e$-health que promueve el desarrollo saludable en la primera infancia, aportando conocimientos y experiencias a las familias sobre la crianza y la educación en los ámbitos de la vinculación afectiva, la alimentación, el juego y el sueño. Este programa está siendo ampliamente utilizado por las figuras parentales y por los profesionales, registrando en ambos casos altos niveles de satisfacción ${ }^{(3)}$. Con el objetivo de garantizar el uso universal de este programa, la Dirección General de Salud Pública del Gobierno de Canarias, en el marco de la aplicación de la estrategia en su comunidad, ha impulsado un estudio piloto en el que se combina el uso de dicho programa online con el apoyo parental presencial, tanto en talleres grupales como en la consulta pediátrica, en los centros de atención primaria, para comprobar así su efectividad. El presente trabajo forma parte de este estudio, aportando la validación de un instrumento de evaluación de la satisfacción parental del apoyo recibido por el equipo de atención pediátrica.

La nueva visión del apoyo parental en el ámbito sanitario sigue el Modelo Centrado en la Familia (en adelante, MCF), basado en establecer una relación de colaboración entre los profesionales y la familia, que se ofrece desde la dignidad y el respeto, en la que se comparten tanto la información como la responsabilidad de la salud del/la niño/a $\mathbf{a}^{(4)}$. El concepto de apoyo parental está basado en la Recomendación del Consejo de Europa (Rec2006/19)(5) a sus estados miembros sobre Políticas de Apoyo al ejercicio de la Parentalidad Positiva. En esta, se señala la responsabilidad del Estado para brindar apoyo parental mediante programas basados en la evidencia dirigidos a la familia, tanto en los ámbitos educativos y sociales como en el ámbito sanitario, para garantizar de esta manera los derechos de la infancia y promover su bienestar personal y social.

La incorporación de la familia en general, y de las figuras parentales en particular, como agentes promotores de la salud a los/as niños/as entre 0 y 3 años responde también a la evidencia científica que ha venido señalando de forma consistente que la familia es un agente clave en la salud y el desarrollo del/la niño/a $\mathbf{a}^{(6,7,8)}$. Además, la inclusión de la familia en la atención pediátrica aporta información relevante sobre cómo promover la salud del/la niño/a en el entorno más cercano ${ }^{(9,10)}$. Igualmente, incrementa la adherencia a los tratamientos y a las orientaciones pediátricas, ya que facilita que la familia asuma activamente su rol como agente valedor del desarrollo de sus hijos/as ${ }^{(11)}$.

La evaluación de la satisfacción familiar en el Modelo Centrado en la Familia y desde la Parentalidad Positiva. Como resultado de la introducción de nuevas formas de actuación en la práctica pediátrica, los estándares de calidad en los servicios están cambiando. De ello se deriva la necesidad de actualizar los instrumentos de evaluación de los servicios de atención primaria. De acuerdo con el MCF y el marco de la Parentalidad Positiva, la evaluación de la calidad de la atención prestada en el servicio debe considerar la voz de las familias como un recurso muy útil para la planificación y mejora 
de las intervenciones basadas en la evidencia. En este sentido, la satisfacción ha sido considerada como el constructo principal para incorporar el punto de vista de la familia ${ }^{(12)}$, ya que constituye un indicador central de la calidad del servicio $^{(13)}$. Además, la satisfacción se relaciona con variables relevantes como la asistencia a las citas programadas o la adherencia al tratamiento $^{(14,15)}$. Así mismo, la satisfacción nos brinda información de hasta qué punto las familias sienten que sus necesidades (y las de sus hijos/as) están siendo cubiertas.

Tradicionalmente, la satisfacción se ha medido como una dimensión general y en términos positivos ${ }^{(16,17)}$. Sin embargo, es importante considerar los dos aspectos de la satisfacción general, la percepción de la adecuación del servicio y el descontento con el servicio, como dimensiones positivas y negativas diferenciadas. De este modo, se evita la mera acumulación de valoraciones positivas y negativas que no ofrecen una imagen contrastada de las fortalezas y debilidades del servicio.

Además de la satisfacción general, es necesario incluir indicadores de satisfacción que se relacionen con los componentes centrales del MCF y del marco de la Parentalidad Positiva. En el caso del MCF, es crucial evaluar la relación de colaboración entre el/la profesional y la figura parental para identificar las necesidades específicas de cada familia y poder elaborar conjuntamente una intervención individualizada y ajustada a cada situación $^{(4)}$. Por su parte, en relación con el marco de la Parentalidad Positiva, se debe evaluar en qué medida las actuaciones de los profesionales ayudan a la promoción de las habilidades parentales y su autopercepción positiva, de modo que se garantice una mayor autonomía de funcionamiento de la familia ${ }^{(18)}$.

En el área de la pediatría, los cuestionarios más empleados de satisfacción están en lengua inglesa y han omitido tanto la relación de colaboración como la capacitación de las familias como factores principales (por ejemplo, Parent's Perceptions of Primary Care $^{(19)}$, Child ZAP(20), Parents' Perceptions of Satisfaction with Care from Pediatric Nurse Practitioners $\left.^{(21)}\right)$. Además, los instrumentos se han desarrollado para evaluar programas específicos $^{(22)}$, unidades de cuidados intensivos ${ }^{(23)} \mathrm{O}$ atención hospitalaria ${ }^{(17,24)}$. Por tanto, la elaboración de un instrumento en español para evaluar la satisfacción parental en atención primaria desde esta nueva perspectiva puede cubrir un hueco muy importante.

El objetivo de este estudio fue desarrollar y evaluar la fiabilidad y validez de constructo del cuestionario "Satisfacción con el Apoyo Parental recibido en Atención Primaria" (en adelante, SATAP-AP) (anexo I).

\section{SUJETOS Y MÉTODOS}

Participantes. Los participantes fueron 226 figuras parentales con hijos/as entre 0 y 36 meses, usuarias de la atención pediátrica de los centros de Salud de la isla de Tenerife y de Gran Canaria. Todos ellos formaron parte de un estudio más amplio que evaluó el programa de apoyo parental basado en el curso online "Ganar Salud y Bienestar de cero a tres años", cuya recogida de datos tuvo lugar entre los años 2018 y 2019. Los participantes fueron principalmente madres $(88,1 \%)$, con hijos e hijas en torno al año de edad, con empleo $(76,2 \%)$, los niveles de estudios correspondieron tanto a estudios universitarios $(53,7 \%)$ como a niveles educativos inferiores $(46,3 \%)$, y la mayoría vivían formando familias biparentales (tabla 1).

\section{Instrumentos.}

Desarrollo del cuestionario SATAP-AP. El cuestionario fue elaborado por un panel de tres 


\section{Tabla 1}

Perfil sociodemográfico familiar y percepción de salud del/la niño/a.

\begin{tabular}{|c|c|c|}
\hline \multirow{2}{*}{\multicolumn{2}{|c|}{ Variables }} & $\mathrm{N}=226$ \\
\hline & & $\mathrm{M}(\mathrm{DT}) / \%$ \\
\hline \multicolumn{2}{|c|}{ Edad del adulto (en años). } & $34,41(5,31)$ \\
\hline \multicolumn{2}{|c|}{ Género figura parental. Mujer. } & 88,1 \\
\hline \multicolumn{2}{|c|}{ Edad del niño/a (en meses). } & $12,38(10,17)$ \\
\hline \multicolumn{2}{|c|}{ Género del niño/a. Mujer. } & 46,1 \\
\hline \multicolumn{2}{|l|}{ Primer hijo/a. Sí. } & 67,7 \\
\hline \multicolumn{2}{|c|}{ Percepción de estado de salud del hijo/a (1-5.) } & $4,42(0,69)$ \\
\hline \multirow{3}{*}{ Nivel educativo } & Sin estudios/Estudios secundarios. & 18,5 \\
\hline & Formación profesional. & 27,8 \\
\hline & Estudios universitarios. & 53,7 \\
\hline \multicolumn{2}{|c|}{ Situación laboral. Activo. } & 76,2 \\
\hline \multicolumn{2}{|c|}{ Área de residencia. Urbana. } & 48,9 \\
\hline \multicolumn{2}{|c|}{ Estructura familiar. Biparental. } & 85,9 \\
\hline
\end{tabular}

investigadores expertos del Departamento de Psicología Evolutiva y de la Educación de la Universidad de La Laguna. Las dimensiones del constructo de satisfacción que se pretendieron medir fueron "adecuación del servicio", "descontento con el servicio", "relación de colaboración con la familia" y "capacitación parental”. Como primer paso, se realizó una revisión de los instrumentos en inglés y en español utilizados para evaluar la satisfacción con el servicio, empleando las siguientes palabras clave en la estrategia de búsqueda: "satisfaction" AND "parent*" OR "mother" AND "paediatric*". Los instrumentos identificados evaluaban con mayor frecuencia las siguientes dimensiones: "satisfacción general", "competencia profesional clínica", "competencias transversales" (por ejemplo, comunicación y empatía) y, en menor medida, la "atención centrada en la familia" ${ }^{\prime}(16,17,20,21,25)$.

Como segundo paso, se elaboró una primera versión de 17 ítems con un formato de respuesta de escala tipo Likert (de 1 a 4 puntos), que recogía el grado de acuerdo con las afirmaciones presentadas.. Dicha versión contó con nueve ítems extraídos del Family Feedback CWS questionnaire $^{(26)}$, relativos sobre todo a las áreas de satisfacción general y colaboración con la familia. Además, se elaboraron ocho ítems para evaluar de forma completa el área de colaboración y el de capacitación, ya que no contábamos con 
ejemplos de ítems apropiados en cuestionarios previos. Como último paso antes de la administración del cuestionario, la versión inicial fue revisada por otro grupo de colaboradores expertos para asegurar que la redacción de las instrucciones y de los ítems fuera comprensible, a partir de la cual se realizaron cambios menores en las instrucciones iniciales y en el formato de presentación.

Perfil sociodemográfico. Compuesto ad hoc por 13 preguntas, incluido género y edad de adultos y niño/a, nivel educativo, situación laboral, estructura familiar y área de residencia.

Escala de percepción de salud del/la niño/a $(\mathrm{H}-\mathrm{HANES})^{(27)}$. Las figuras parentales evaluaron en un solo ítem el estado de salud de su hijo/a en una escala de cinco puntos, con una calificación de 1 que indicaba una salud "Muy mala" y 5 para "Muy buena".

Procedimiento. Los participantes fueron captados por los equipos de pediatría de atención primaria en las sesiones de revisión del Programa de Salud Infantil(28), y tenían la opción de completar el cuestionario de forma online o presencial. En ambos casos, se aseguró la confidencialidad y se contó con el consentimiento informado de las familias de forma escrita, de acuerdo con el Comité Ético de la Universidad de La Laguna.

Plan de análisis. Se llevaron a cabo dos procedimientos para evaluar la validez de constructo: un análisis de la estructura factorial así como de la fiabilidad de los factores resultantes, y un análisis de las diferencias individuales en los factores obtenidos en función de las variables sociodemográficas. Para determinar la estructura factorial del cuestionario se utilizó el modelo de ecuaciones estructurales exploratorio $(\mathrm{MESE})^{(29)}$, que combina el análisis factorial exploratorio (AFE) con el análisis factorial confirmatorio (AFC). Como método de estimación se utilizó el de mínimos cuadrados ponderados ajustado por la media y la varianza (WLSMW, Weighted Least Squares Mean and Variance Adjusted). Para analizar el ajuste del modelo al patrón de los datos se tomaron como indicadores el índice de ajuste comparativo (CFI), el índice de bondad de ajuste (GFI), el error cuadrático medio de aproximación (RMSEA) y la raíz del error cuadrático medio (SRMR), tomando en consideración que los parámetros de un buen modelo son: RMSEA < 0,05; CFI y TLI > 0,95; SRMR $<0,1^{(30)}$. Además, se utilizó el test de esfericidad de Bartlett y la medida de adecuación muestral de Kaiser-Mayer-Olikin ${ }^{(31)}$. A continuación, se realizó un análisis de ítems y se evaluó la consistencia interna con eliminación del ítem, utilizando el índice Alfa de Cronbach. Por último, para el análisis de las diferencias individuales, se emplearon ANOVAs con la puntuación tipificada de cada individuo obtenida en cada factor, que refleja la contribución ponderada de cada ítem, para explorar la variabilidad de la satisfacción con el servicio en función del perfil sociodemográfico. Para los contrastes post hoc, se utilizó la prueba Scheffe. Para realizar los ANOVAs y estimar la fiabilidad se utilizó el programa estadístico SPSS 25, y para el MESE, el Mplus 6.11.

\section{RESULTADOS}

Análisis factorial del cuestionario SATAP-AP. El Análisis Confirmatorio de la versión inicial no mostró índices apropiados para ninguna estructura factorial, de acuerdo con los criterios de Tabachnick \& Fidell ${ }^{(30)}$. Tras la eliminación de dos ítems, la estructura de cuatro factores mostró unos índices de ajuste adecuados, por lo que la versión resultante fue de 15 ítems (tabla 2).

A continuación, se muestra la estructura factorial final con los 14 ítems significativos que la componen (el ítem "Los/as profesionales resuelven mis dudas dándome toda la información que necesito" fue eliminado ya que no resultó 
significativo en ningún factor), el orden en que se presentaron los ítems y sus pesos factoriales (figura 1):

- F1: "Adecuación del servicio", con cuatro ítems.

- F2: “Colaboración con la familia", con tres ítems.

- F3: "Descontento con el servicio", con cuatro ítems, y "Capacitación parental", con tres ítems.

- F4: "Capacitación parental", con cuatro ítems.
En la tabla 3 se presentan todos los pesos factoriales de la versión final según sus factores correspondientes.

En el análisis de la consistencia interna global de las sub-escalas, ninguno de los elementos reveló un comportamiento inapropiado (tabla 4). Las medias indicaron estar "bastante de acuerdo" con aquellos ítems con contenidos positivos relativos a los factores $1,2 \mathrm{y}$ 4, y "poco de acuerdo" con aquellos ítems con contenidos negativos que indicaban "descontento con el servicio" relativos al factor 3 . Las desviaciones estándar alcanzaron valores cercanos a 1,00 , por lo que fue posible asumir una variabilidad suficiente en las puntuaciones

Tabla 2

Índices de ajuste de los modelos del análisis factorial confirmatorio ( $\mathrm{N}=\mathbf{2 2 6})$.

\begin{tabular}{|l|c|c|c|c|}
\hline \multicolumn{1}{|c|}{ Factores } & RMSEA & CFI & TLI & SRMR \\
\hline 3 factores & 0,07 & 0,96 & 0,94 & 0,03 \\
\hline 4 factores & 0,03 & 0,99 & 0,99 & 0,02 \\
\hline 5 factores & - & - & - & - \\
\hline
\end{tabular}

RMSEA: Root Mean Square Error of Approximation; CFI: Comparative Fit Index; TLI Tucker-Lewis Index; SRMR: Standarized Root Mean-Square.

\section{Figura 1}

Diagrama del modelo mostrando los factores, ítems y pesos factoriales.

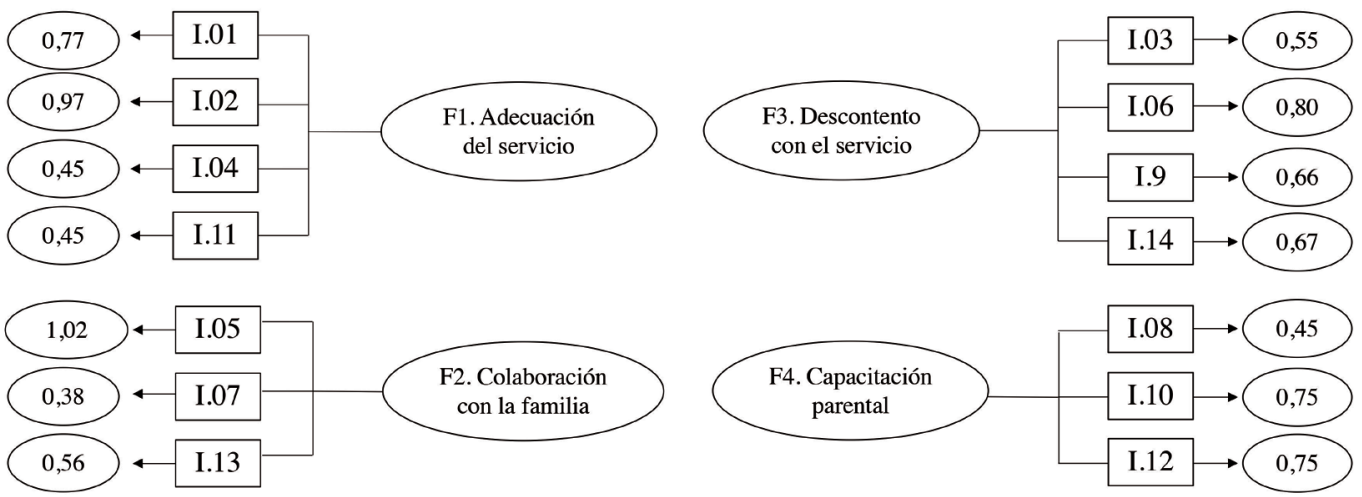




\section{Tabla 3}

Pesos factoriales para la versión definitiva de la escala según el modelo estandarizado para los 4 factores.

\begin{tabular}{|c|c|c|c|c|}
\hline \multirow[b]{2}{*}{ Ítems SATAP-AP } & \multicolumn{4}{|c|}{ Factores } \\
\hline & $\begin{array}{l}\text { F1. } \\
\text { Adecuación } \\
\text { del servicio }\end{array}$ & \begin{tabular}{|c|} 
F2. \\
Colaboración \\
con la \\
familia
\end{tabular} & \begin{tabular}{|c|} 
F3. \\
Descontento \\
con el \\
servicio
\end{tabular} & $\begin{array}{c}\mathrm{F} 4 . \\
\text { Capacitación } \\
\text { parental }\end{array}$ \\
\hline $\begin{array}{l}\text { 1. Estoy satisfecho/a con los servicios } \\
\text { que me ofrecen en este centro. }\end{array}$ & $\mathbf{0}, 77^{(* *)}$ & 0,05 & $-0,02$ & 0,02 \\
\hline $\begin{array}{l}\text { 2. En general, estoy satisfecho/a con el apoyo } \\
\text { que recibimos en la consulta. }\end{array}$ & $\mathbf{0 , 9 7} 7^{(* * *)}$ & $-0,05$ & $-0,01$ & 0,01 \\
\hline $\begin{array}{l}\text { 4. La ayuda que recibo aquí es mejor de lo } \\
\text { que esperaba. }\end{array}$ & $\mathbf{0 , 4 5} 5^{(* *)}$ & $0,33^{(* *)}$ & 0,00 & 0,13 \\
\hline $\begin{array}{l}\text { 11. Estoy de acuerdo con lo que los/as profesionales } \\
\text { creen mejor para mi hijo/a. }\end{array}$ & $\mathbf{0 , 4 5 ^ { ( * * ) }}$ & 0,09 & 0,05 & 0,29 \\
\hline $\begin{array}{l}\text { 5. Aquí he aprendido mucho sobre cómo fomentar } \\
\text { los hábitos saludables en mi hijo/a. }\end{array}$ & $-0,02$ & $\mathbf{1 , 0 2} 2^{(* * *)}$ & 0,01 & $-0,02$ \\
\hline 7. Los/as profesionales entienden cómo me siento. & 0,15 & $\mathbf{0 , 3 8 ^ { ( * * ) }}$ & $-0,07$ & $0,35^{(* *)}$ \\
\hline $\begin{array}{l}\text { 13. En este centro me han enseñado a buscar } \\
\text { y conseguir ayuda en otros sitios. }\end{array}$ & 0,18 & $\mathbf{0 , 5 6} 6^{(* * *)}$ & 0,00 & 0,07 \\
\hline $\begin{array}{l}\text { 3. Mi hijo/a no está recibiendo el tipo de ayuda } \\
\text { que necesita. }\end{array}$ & $-0,02$ & $-0,07$ & $\mathbf{0 , 5 5} 5^{(* *)}$ & 0,26 \\
\hline $\begin{array}{l}\text { 6. Siento que la atención que estoy recibiendo } \\
\text { aquí no es útil. }\end{array}$ & 0,14 & 0,01 & $\mathbf{0}, \mathbf{8 0}(* * *)$ & $-0,11$ \\
\hline $\begin{array}{l}\text { 9. Los/as profesionales me cuestionan mis } \\
\text { decisiones sobre mi hijo/a. }\end{array}$ & $-0,02$ & $-0,02$ & $\mathbf{0 , 6 6} 6^{(* *)}$ & 0,02 \\
\hline $\begin{array}{l}\text { 14. Los/as profesionales no tienen tiempo } \\
\text { de escuchar. }\end{array}$ & $-0,01$ & 0,13 & $\mathbf{0 , 6 7} 7^{(* * *)}$ & $-0,00$ \\
\hline $\begin{array}{l}\text { 8. Sé que lo que cuento a los/as profesionales } \\
\text { no saldrá de aquí. }\end{array}$ & 0,28 & $-0,01$ & $-0,02$ & $0,45^{(* * *)}$ \\
\hline $\begin{array}{l}\text { 10. Los/as profesionales tienen en cuenta mi } \\
\text { opinión a la hora de decidir sobre mi hijo/a. }\end{array}$ & $-0,01$ & 0,02 & $-0,10$ & $\mathbf{0 , 7 5 ^ { ( * * * ) }}$ \\
\hline $\begin{array}{l}\text { 12. Los/as profesionales me hacen sentir } \\
\text { capacitado/a como padre/madre. }\end{array}$ & 0,13 & 0,06 & 0,05 & $\mathbf{0}, 75^{(* * *)}$ \\
\hline
\end{tabular}


Tabla 4

Media (M), Desviación Típica (DT) y Alfa de Cronbach si se elimina el ítem de cada factor (a-i).

\begin{tabular}{|c|c|c|c|c|}
\hline Factores & Ítems & M & DT & $a-i$ \\
\hline \multirow{4}{*}{$\begin{array}{l}\text { F1. Adecuación del servicio } \\
\alpha=0,90\end{array}$} & 1 & 3,18 & 0,80 & 0,88 \\
\hline & 2 & 3,30 & 0,85 & 0,85 \\
\hline & 4 & 3,05 & 0,90 & 0,87 \\
\hline & 11 & 3,09 & 0,84 & 0,89 \\
\hline \multirow{3}{*}{$\begin{array}{l}\text { F2. Colaboración con la familia } \\
\alpha=0,89\end{array}$} & 5 & 2,90 & 0,97 & 0,83 \\
\hline & 7 & 2,93 & 0,87 & 0,84 \\
\hline & 13 & 2,75 & 1,01 & 0,88 \\
\hline \multirow{4}{*}{$\begin{array}{l}\text { F3. Descontento con el servicio } \\
\alpha=0,83\end{array}$} & 3 & 2,36 & 1,24 & 0,83 \\
\hline & 6 & 2,10 & 1,21 & 0,73 \\
\hline & 9 & 2,26 & 1,12 & 0,81 \\
\hline & 14 & 2,04 & 1,13 & 0,77 \\
\hline \multirow{3}{*}{$\begin{array}{l}\text { F4. Capacitación parental } \\
\alpha=0,85\end{array}$} & 8 & 3,38 & 0,77 & 0,86 \\
\hline & 10 & 3,14 & 0,80 & 0,76 \\
\hline & 12 & 3,25 & 0,75 & 0,73 \\
\hline
\end{tabular}

de acuerdo con los supuestos ${ }^{(29)}$. Las sub-escalas mostraron una fiabilidad de entre $0,83 \mathrm{y}$ 0,90 , alcanzando un cociente de fiabilidad total de $\alpha=0,84$.

Análisis de las diferencias individuales en función de las variables sociodemográficas y la salud infantil. Solo las variables sociodemográficas mostraron diferencias significativas en los factores. En la tabla 5 se recogen aquellas variables que mostraron diferencias significativas en al menos un factor. En relación con el factor "Adecuación del servicio", se observaron diferencias significativas entre los diferentes niveles educativos. Aquellos participantes sin estudios o con estudios secundarios mostraron mayor satisfacción que aquellos con estudios universitarios o con formación profesional. Además, la estructura familiar resultó significativa, ya que las familias biparentales mostraron más satisfacción que las monoparentales (tabla 5).
El factor "Colaboración con la familia" mostró mayor variabilidad en función de las características sociodemográficas. Específicamente, se observaron diferencias significativas en relación con el nivel educativo. Los participantes con menor nivel de estudios (Sin estudios/ Estudios secundarios) percibieron niveles más altos de colaboración del/la profesional con la familia que aquellos con formación profesional o estudios universitarios. Los participantes en situación de desempleo reportaron mayores niveles de colaboración que aquellos en situación activa. Además, las familias monoparentales percibieron menos colaboración del/la profesional que las biparentales (tabla 5).

En el factor de "Capacitación parental" de nuevo resultó significativo el nivel de estudios. Los participantes con menor nivel educativo (Sin estudios/Estudios secundarios) percibieron que 


\section{Tabla 5}

Diferencias individuales en cada factor del cuestionario SATAP-AP en función de las variables sociodemográficas.

\begin{tabular}{|c|c|c|c|c|}
\hline \multirow[t]{2}{*}{ Variables } & $\begin{array}{l}\text { F1. } \\
\text { Adecuación } \\
\text { con el } \\
\text { servicio }\end{array}$ & $\begin{array}{l}\text { F2. } \\
\text { Colaboración } \\
\text { con la } \\
\text { familia }\end{array}$ & $\begin{array}{l}\text { F3. } \\
\text { Descontento } \\
\text { con el } \\
\text { servicio }\end{array}$ & $\begin{array}{l}\text { F4. } \\
\text { Capacitación } \\
\text { parental }\end{array}$ \\
\hline & $\mathrm{M}(\mathrm{DT})$ & M (DT) & M (DT) & M (DT) \\
\hline \multirow{2}{*}{ Nivel educativo } & $\mathbf{F}(2,218)=6,55^{(* *)}$ & $\mathrm{F}(2,218)=6,88^{(* * *)}$ & $F(1,218)=2,15$ & $\mathrm{~F}(2,218)=6,29^{(*)}$ \\
\hline & $\begin{array}{c}\text { Post hoc } \\
\mathbf{1 - 2}^{(* *)}, \mathbf{1 - 3}^{*}, 2-3\end{array}$ & $\begin{array}{c}\text { Post hoc } \\
\mathbf{1 - 2}^{(* *)}, \mathbf{1 - 3}^{(* *)}, 2-3\end{array}$ & $\begin{array}{c}\text { Post hoc } \\
1-2,1-3,2-3\end{array}$ & $\begin{array}{c}\text { Post hoc } \\
\mathbf{1 - 2}^{(* *)}, \mathbf{1 - 3 ^ { ( * * ) }}, 2-3\end{array}$ \\
\hline $\begin{array}{l}\text { Sin estudios / Estudios } \\
\text { secundarios }\end{array}$ & $0,46(0,70)$ & $0,52(0,80)$ & $-0,04(0,89)$ & $0,45(0,73)$ \\
\hline Formación profesional & $-0,25(-1,07)$ & $-0,12(1,02)$ & $-0,18(0,74)$ & $-0,18(1,00)$ \\
\hline Estudios universitarios & $-0,01(0,94)$ & $-0,11(0,98)$ & $0,11(0,96)$ & $-0,05(0,93)$ \\
\hline Situación laboral & $F(1,219)=3,20$ & $\mathrm{~F}(1,219)=3,78^{(*)}$ & $F(1,219)=0,95$ & $\mathrm{~F}(1,219)=1,81$ \\
\hline Activo & $-0,07(0,98)$ & $-0,05(1,02)$ & $0,03(0,91)$ & $-0,05(0,96)$ \\
\hline Desempleado & $0,20(0,92)$ & $0,22(0,86)$ & $-0,10(0,86)$ & $0,14(0,85)$ \\
\hline Estructura familiar & $\mathrm{F}(1,219)=4,55^{(*)}$ & $F(1,219)=5,80^{(*)}$ & $\mathrm{F}(1,219)=1,29$ & $F(1,219)=1,06$ \\
\hline Monoparental & $-0,34(1,19)$ & $-0,39(1,10)$ & $0,17(1,01)$ & $-0,16(1,03)$ \\
\hline Biparental & $-0,06(0,94)$ & $0,06(0,96)$ & $-0,03(0,88)$ & $0,03(0,92)$ \\
\hline \multicolumn{5}{|c|}{$\begin{array}{l}(*) \mathrm{p}<0,05 ;(* *) \mathrm{p}<0,01 ;(* * *) \mathrm{p}<0,001 ; \mathrm{M}: \text { Media; DT: Desviación Típica; F: Prueba } \mathrm{F} \text { de Fisher. } \\
\text { Nivel educativo codificado como } 1=\text { Sin estudios/Estudios secundarios, } 2=\text { Formación profesional y } \\
3=\text { Estudios universitarios. }\end{array}$} \\
\hline
\end{tabular}

el apoyo recibido promovía más sus capacidades que aquellos con formación profesional o con estudios universitarios (tabla 5).

\section{DISCUSIÓN}

Este estudio tiene por objetivo evaluar la validez de constructo y la fiabilidad del cuestionario SATAP-AP, así como la variabilidad de sus puntuaciones en función del perfil sociodemográfico y la salud infantil percibida. Los resultados muestran que el modelo más adecuado es el conformado por cuatro factores, ya que es el que ofrece un número óptimo de dimensiones subyacentes al constructo de apoyo parental en el ámbito sanitario, teniendo en cuenta la posible complejidad del mismo. Así, se obtienen dos factores que dan cobertura a la satisfacción general con su extremo positivo y negativo bien diferenciados. El Factor 1, "Adecuación del servicio", aporta información sobre hasta qué punto la familia percibe que sus necesidades y expectativas se ven cubiertas y satisfechas con la atención recibida, así 
como se muestran de acuerdo con lo que el/la profesional cree mejor para su hijo/a. Por su parte, el Factor 3, "Descontento con el servicio", manifiesta el grado de insatisfacción que el usuario siente debido a su desconfianza respecto al tipo de ayuda que prestan a su hijo/a, sobre la perspectiva crítica de los profesionales hacia sus decisiones como padres/madres y el escaso tiempo que éstos dedican a escucharles, tres aspectos claves que sin duda van a entorpecer el proceso de apoyo a las familias.

Los otros dos factores obtenidos reflejan dos dimensiones positivas del constructo de apoyo parental. El Factor 2, "Colaboración con la familia", define muy bien una de las características del MCF, al enfatizar la importancia de establecer relaciones de colaboración entre el/ la profesional y la familia ${ }^{(4,32)}$ que, a menudo, se circunscriben a recomendaciones y orientaciones unidireccionales que provienen del/la profesional. Los contenidos de este factor hacen referencia a las oportunidades de aprendizaje recibidas sobre cómo fomentar los hábitos saludables en su hijo/a, a la empatía mostrada por el/la profesional sobre cómo se sienten las familias y a la apertura de redes de apoyo que pueden complementar el que reciben del servicio. La percepción de colaboración es clave, ya que posibilita que las figuras parentales se sientan más autorizadas para corresponsabilizarse sobre la promoción de la salud de su hijo/a.

El Factor 4, “Capacitación parental”, está basado en el marco de la Parentalidad Positiva ${ }^{(33)}$, destacando tres aspectos muy relevantes en el ámbito sanitario: la confidencialidad de lo compartido con la familia, la actitud de valorar y tener en cuenta el punto de vista de los padres/ madres a la hora de la toma de decisiones ${ }^{(32)}$, y promover una percepción positiva de sus capacidades parentales, siendo también la autoeficacia un aspecto clave en el $\mathrm{MCF}^{(34)}$ y como factor protector de la depresión posnatal en el caso de las madres ${ }^{(35)}$. Todo ello incrementa su autoconfianza y les da mayor seguridad y autonomía cuando cuidan o educan a sus hijos/as.

En relación con la aplicación y corrección del cuestionario, recomendamos la estructura propuesta ya que respeta las cargas factoriales significativas en cada factor con las siguientes particularidades. En cuanto al ítem 4 ("La ayuda que recibo aquí es mejor de lo que esperaba"), a pesar de tener una carga significativa en los factores 1 y 2, "Adecuación del servicio" y "Colaboración con la familia", recomendamos integrarlo en el cálculo del primer factor por ser el que presenta un carga mayor y mayor ajuste a su contenido. Por su parte, el ítem 7 ("Los/as profesionales entienden cómo me siento") muestra cargas factoriales significativas similares en los factores $2 \mathrm{y}$ 4, "Colaboración con la familia" y "Capacitación parental”. No obstante, por su relación con la empatía que se hace indispensable en toda colaboración, recomendamos asociarlo al Factor 2. Para el uso práctico del cuestionario recomendamos calcular la media de los ítems en cada factor.

Con respecto a la variabilidad de las puntuaciones del cuestionario en función de las variables sociodemográficas, se encuentra que aquellas figuras parentales con mayor nivel educativo muestran menores niveles de satisfacción, excepto en el caso del "Descontento con el servicio", que no resulta sensible a ninguna variable del perfil. En este sentido, diferentes estudios señalan que los pacientes con menor nivel educativo tienden a estar más satisfechos con la atención médica que reciben, probablemente porque sus estándares de exigencia son menos altos ${ }^{(34,35,36)}$. Esta variabilidad, además de reforzar la validez de constructo, hace del instrumento una herramienta útil para aplicar a la población general y evaluar acciones de prevención universal ${ }^{(37,38)}$. Igualmente, la situación de desempleo, probablemente por su alta relación con el nivel de estudios, se asocia a niveles más altos de satisfacción en el factor "Colaboración con la familia"(39). 
En cuanto a la estructura familiar, el hecho de que las familias monoparentales se muestren menos satisfechas en el factor de "Colaboración con la familia" puede explicarse porque tienen un conjunto más amplio de necesidades, ya que deben compatibilizar las demandas laborales con el cuidado del niño o de la niña ${ }^{(40)}$, necesidades que pueden no verse satisfechas en el limitado espacio de la consulta de atención primaria. No obstante, los estudios aún arrojan datos contradictorios en cuanto a la relación entre la estructura familiar y la satisfacción con el servicio ${ }^{(39)}$. Por último, que las familias primíparas y no primíparas no muestren diferencias significativas refleja que las necesidades de apoyo se dan de forma continuada en la crianza. Además, estas se dan igualmente con independencia del estado de salud percibido.

Este estudio presenta algunas limitaciones. La primera es haber contado sobre todo con la madre como informante, sin haber podido darle mayor presencia al punto de vista del padre, aunque refleja una realidad actual, ya que la asistencia al pediatra y el cuidado de la infancia gravita todavía en mayor medida sobre la madre. La segunda limitación es no haber recogido más medidas que permitiesen evaluar otros tipos de validez como la concurrente o la predictiva. La tercera limitación está relacionada con las edades de los/as hijos/as, que solo abarcaron la primera etapa de la infancia. En futuras investigaciones sería interesante comprobar si es necesario ajustar el cuestionario para edades posteriores donde las necesidades de las figuras parentales pueden variar (por ejemplo, segunda infancia, preadolescencia).

En conclusión, el cuestionario SATAP-AP, a pesar de su brevedad, permite una evaluación completa y fiable de la satisfacción de las familias con el apoyo parental recibido en el servicio de pediatría de atención primaria. Todo ello hace del SATAP-AP un recurso útil para la mejora continua del servicio hacia las buenas prácticas profesionales recomendadas en el MCF y en el enfoque de la Parentalidad Positiva, ya que evalúa facetas importantes del apoyo parental que resultan claves en dicho ámbito sanitario.

\section{BIBLIOGRAFÍA}

1. Ministerio de Sanidad Servicios Sociales e Igualdad. Estrategia de Promoción de La Salud y Prevención En El Sistema Nacional de Salud. (Dirección General de Salud Pública, Calidad e Innovación, ed.). Madrid, España; 2013.

2. Rodrigo MJ, Martín-Quintana JC, Máiquez ML et al. Parentalidad Positiva: Ganar Salud y Bienestar de 0-3 Años Guía Para El Desarrollo de Talleres Presenciales Grupales Coordinación Técnica Institucional. Madrid, España: Ministerio de Sanidad Servicios Sociales e Igualdad; 2017.

3. Callejas E, Byrne S, Rodrigo MJ. "Gaining health and wellbeing from birth to three": a web-based positive parenting programme for primary care settings. Early Child Development and Care. 2018;188(11):1552-1565. doi:10.1 080/03004430.2018.1490896.

4. Stille C, Turchi RM, Antonelli R et al. The family-centered medical home: Specific considerations for child health Research and policy. Academic Pediatrics. 2010;10(4):211217. doi:10.1016/j.acap.2010.05.002.

5. Consejo de Europa. Recomendación Rec (2006)19 Del Comité de Ministros a Los Estados Miembros Sobre Políticas de Apoyo a La Parentalidad Positiva.; 2006. https://www.mscbs.gob.es/ssi/familiasInfancia/parentalidadPos2012/docs/informeRecomendacion.pdf.

6. Shonkoff JP, Fisher PA. Rethinking evidence-based practice and two-generation programs to create the future of early childhood policy. Development and Psychopathology. 2013;24(6188):1635-1653. doi:10.1017/ S0954579413000813.

7. Guralnick MJ. Developmental Science and Preventive Interventions for Children at Environmental Risk. Infants 
\& Young Children. 2013;26(4):270-285. doi:10.1097/ IYC.0b013e3182a6832f.

8. Shah R, Kennedy S, Clark MD, Bauer SC, Schwartz A. Primary Care-Based Interventions to Promote Positive Parenting Behaviors: A Meta-analysis. Pediatrics. 2016;137(5):e20153393e20153393. doi:10.1542/peds.2015-3393.

9. Zvara BJ, Schoppe-Sullivan SJ, Dush CK. Fathers' involvement in child health care: Associations with prenatal involvement, parents' beliefs, and maternal gatekeeping. Family Relations. 2013;62(4):649-661. doi:10.1111/ fare. 12023 .

10. Daly M, Bray R, Bruckauf Z et al. Family and Parenting Support Policy and Provision in a Global Context Policy and Provision in a Global Context. Florencia: Innocenti Insight; 2015.

11. Han E, Genevro J. Engaging patients and families in the medical home. AHRQ Publication. 2010;(10):1-73. doi:AHRQ Publication No. 10-0083-E.

12. Ayala-Nunes L, Jiménez L, Hidalgo V, Jesus S. Family feedback in Child Welfare Services: A systematic review of measures. Children and Youth Services Review. 2014;44:299-306. doi:10.1016/j.childyouth.2014.07.004.

13. Ruiz-Moral R, Pérula de Torres LA, Muñoz Alamo M et al. Consultas de medicina de familia: comparación de tres métodos para investigar necesidades no satisfechas. Revista Española Salud Publica. 2011;85(3):315-322.

14. Niet J De, Timman R, Jongejan M, Passchier J. Predictors of Participant Dropout at Various Stages of a Pediatric Lifestyle Program. Pediatrics. 2011;127:164-170. doi:10.1542/peds.2010-0272.

15. Skelton J, Irby MB, Geiger A. A Systematic Review of Satisfaction and Pediatric Obesity Treatment: New Avenues for Addressing Attrition. Journal for Healthcare Quality. 2014;36(4):5-22. doi:10.1111/jhq.12003.

16. Weissenstein A, Straeter A, Villalon G, Luchter E, Bittmann S. Parent satisfaction with a pediatric practice in
Germany : A questionnaire-based study. Italian Journal of Pediatrics. 2011;37(1):31. doi:10.1186/1824-7288-37-31.

17. Schmidt S, Thyen U, Chaplin J, Mueller-Godejfroy E. Process of Instrument Development. Ambulatory Pediatrics. 2007;7(5):374-383.

18. Rodrigo MJ, Byrne S, Alvarez M. Interventions to Promote Positive Parenting in Spain. In: Romano J., Israelashvili M, eds. The Cambridge Handbook of International Prevention Science. Cambridge: Cambridge University Press; 2016:929956. doi:10.1017/9781316104453.040.

19. Seid M, Varni JW. Measuring primary care for children of Latino farmworkers: Reliability and validity of the parent's perceptions of primary care measure (P3C). Maternal and Child Health Journal. 2005;9(1):49-57. doi:10.1007/s10995-005-2450-8.

20. Bitzer EM, Volkmer S, Petrucci M, Weissenrieder N, Dierks M. Patient satisfaction in pediatric outpatient settings from the parents perspective - The Child ZAP : A psychometrically validated standardized questionnaire. BMC Health Services Research. 2012;12(1):1. doi:10.1186/1472-6963-12-347.

21. DiAnna Kinder F, Allen LR. Parents' perception of satisfaction with care from pediatric nurse practitioners instrument. Journal of Pediatric Health Care. 2014;28(2):128135. doi:10.1016/j.pedhc.2012.12.009.

22. Summers JA, Hoffman L, Marquis J, Turnbull A, Poston D. Relationship Between Parent Satisfaction Regarding Partnerships With Professionals and Age of Child. Topics in Early Childhood Special Education. 2005;25(1):48-58. doi:10.1177/02711214050250010501.

23. Latour JM, Duivenvoorden HJ, Tibboel D, Hazelzet JA. The shortened EMpowerment of PArents in THe Intensive Care 30 questionnaire adequately measured parent satisfaction in pediatric intensive care units. Journal of Clinical Epidemiology. 2013;66:1045-1050. doi:10.1016/j.jclinepi.2013.02.010.

24. Hyassat M, Akhayat M, Alzyoud M. Development of a scale for measuring parental satisfaction with services 
available for disabled children in Jordan. International Journal of Special Education. 2015;30(1):37-44.

25. Ireys HT, Perry JJ. Development and Evaluation of a Satisfaction Scale for Parents of Children With Special Health Care Needs. Pediatrics. 1999;104(5):1182-1191. doi: $10.1038 / 343728 \mathrm{a} 0$.

26. Ayala-Nunes L, Jimenez L, Hidalgo V, Dekovic M, Jesus S. Development and Validation of the Family Feedback on Child Welfare Services (FF-CWS). Research on Social Work Practice. 2016;28(2):1-11. doi:10.1177/1049731516642842.

27. Arcia E. Latino parents' perception of their children's health status. Social Science and Medicine. 1998;46(10):12711274. doi:10.1016/S0277-9536(97)10055-7.

28. Servicio Canario de Salud. Programa de Salud Infantil. (Reyes Melián JM, ed.). Las Palmas de Gran Canaria, España: Dirección General de Programas Asistenciales; 2007.

29. Asparouhov T, Muthén B. Exploratory structural equation modeling. Structural Equation Modeling. 2009;16(3):397-438. doi:10.1080/10705510903008204.

30. Tabachnick B, Fidell L. Using Multivariate Statistics. 6th ed. (Pearson, ed.). Boston; 2013.

31. Pérez ER, Medrano L. Análisis factorial exploratorio : Bases conceptuales y metodológicas. Revista Argentina de Ciencias del Comportamiento. 2010;2(1889):58-66.

32. Kuo DZ, Houtrow AJ, Arango P, Kuhlthau KA, Simmons JM, Neff JM. Family-centered care: Current applications and future directions in pediatric health care. Maternal and Child Health Journal. 2012;16(2):297-305. doi:10.1007/s10995-011-0751-7.

33. Rodrigo MJ, Máiquez ML, Martín JC. Parentalidad positiva y políticas locales de apoyo a las familias. Ministerios de Salud y Politica Social Federación Española de Municipios y Provincias. 2010:7-23. https://www.mscbs. gob.es/ssi/familiasInfancia/docs/folletoParentalidad.pdf.

34. Ulfsdotter M, Enebrink P, Lindberg L. Effectiveness of a universal health-promoting parenting program: A randomized waitlistcontrolled trial of All Children in Focus. BMC Public Health. 2014;14(1):1-11. doi:10.1186/14712458-14-1083.

35. Leahy-Warren P, Mccarthy G, Corcoran P. First-time mothers: Social support, maternal parental self-efficacy and postnatal depression. Journal of Clinical Nursing. 2012;21(34):388-397. doi:10.1111/j.1365-2702.2011.03701.x.

36. Quintana JM, González N, Bilbao A et al. Predictors of patient satisfaction with hospital health care. BMC Health Services Research. 2006;6:1-9. doi:10.1186/1472-6963-6-102.

37. Rahmqvist M, Bara AC. Patient characteristics and quality dimensions related to patient satisfaction. International Journal for Quality in Health Care. 2010;22(2):86-92. doi:10.1093/intqhe/mzq009.

38. Bleich SN, Ozaltin E, Murray CJL. How does satisfaction with the health-care system relate to patient experience? Bulletin of the World Health Organization. 2009;87(4):271-278. doi:10.2471/BLT.07.050401.

39. Batbaatar E, Dorjdagva J, Luvsannyam A, Savino MM, Amenta P. Determinants of patient satisfaction: A systematic review. Perspectives in Public Health. 2017;137(2):89101. doi:10.1177/1757913916634136.

40. Whisenhunt JL, Chang CY, Parrish MS, Carter JR. Addressing Single Parents'Needs in ProfessionalCounseling:A Qualitative Examination of Single Parenthood. Family Journal. 2019;27(2):188-198. doi:10.1177/1066480719835343. 


\section{Anexo I}

Versión final del cuestionario SATAP-AP.

Nos gustaría conocer su satisfacción con el apoyo parental que recibe en su centro de atención primaria en la consulta de pediatría. Por favor, indique su grado de acuerdo con las siguientes afirmaciones.

Nota: 1, Nada de acuerdo; 2, Poco de acuerdo; 3, Bastante de acuerdo; 4, Totalmente de acuerdo.

\begin{tabular}{|c|c|c|c|c|c|}
\hline \multicolumn{2}{|r|}{ Preguntas } & $\begin{array}{c}\text { Nada } \\
\text { de acuerdo } \\
1\end{array}$ & $\begin{array}{c}\begin{array}{c}\text { Poco } \\
\text { de acuerdo }\end{array} \\
2\end{array}$ & $\begin{array}{c}\begin{array}{c}\text { Bastante } \\
\text { de acuerdo }\end{array} \\
3\end{array}$ & $\begin{array}{c}\text { Totalmente } \\
\text { de acuerdo } \\
4\end{array}$ \\
\hline 1 & $\begin{array}{l}\text { Estoy satisfecho/a con los servicios } \\
\text { que me ofrecen en este centro. }\end{array}$ & & & & \\
\hline 2 & $\begin{array}{l}\text { En general, estoy satisfecho/a con } \\
\text { el apoyo que recibimos en la consulta. }\end{array}$ & & & & \\
\hline 3 & $\begin{array}{l}\text { Mi hijo/a no está recibiendo el tipo } \\
\text { de ayuda que necesita. }\end{array}$ & & & & \\
\hline 4 & $\begin{array}{l}\text { La ayuda que recibo aquí es mejor } \\
\text { de lo que esperaba. }\end{array}$ & & & & \\
\hline 5 & $\begin{array}{l}\text { Aquí he aprendido mucho sobre cómo } \\
\text { fomentar los hábitos saludables en } \\
\text { mi hijo/a. }\end{array}$ & & & & \\
\hline 6 & $\begin{array}{l}\text { Siento que la atención que estoy } \\
\text { recibiendo aquí no es útil. }\end{array}$ & & & & \\
\hline 7 & $\begin{array}{l}\text { Los/as profesionales entienden } \\
\text { cómo me siento. }\end{array}$ & & & & \\
\hline 8 & $\begin{array}{l}\text { Sé que lo que cuento a los/as } \\
\text { profesionales no saldrá de aquí. }\end{array}$ & & & & \\
\hline 9 & $\begin{array}{l}\text { Los/as profesionales me cuestionan } \\
\text { mis decisiones sobre mi hijo/a. }\end{array}$ & & & & \\
\hline 10 & $\begin{array}{l}\text { Los/as profesionales tienen en cuenta } \\
\text { mi opinión a la hora de decidir sobre } \\
\text { mi hijo/a. }\end{array}$ & & & & \\
\hline 11 & $\begin{array}{l}\text { Estoy de acuerdo con lo que los/as } \\
\text { profesionales creen mejor para } \\
\text { mi hijo/a. }\end{array}$ & & & & \\
\hline 12 & $\begin{array}{l}\text { Los/as profesionales me hacen sentir } \\
\text { capacitado/a como padre/madre. }\end{array}$ & & & & \\
\hline 13 & $\begin{array}{l}\text { En este centro me han enseñado } \\
\text { a buscar y conseguir ayuda en otros } \\
\text { sitios. }\end{array}$ & & & & \\
\hline 14 & $\begin{array}{l}\text { Los/as profesionales no tienen tiempo } \\
\text { de escuchar. }\end{array}$ & & & & \\
\hline
\end{tabular}

